\title{
Coarsening Dynamics of an Antiferromagnetic $X Y$ model on the Kagome Lattice: Breakdown of the Critical Dynamic Scaling
}

\author{
Sangwoong Park, ${ }^{1}$ Bongsoo Kim, ${ }^{2}$ and Sung Jong Lee ${ }^{1}$ \\ ${ }^{1}$ Department of Physics, The University of Suwon, Kyonggi-do 445-743, Korea \\ ${ }^{2}$ Department of Physics, Changwon National University, Changwon, 641-773, Korea
}

\begin{abstract}
We find a breakdown of the critical dynamic scaling in the coarsening dynamics of an antiferromagnetic $X Y$ model on the kagome lattice when the system is quenched from disordered states into the Kosterlitz-Thouless $(K T)$ phases at low temperatures. There exist multiple growing length scales: the length scales of the average separation between fractional vortices are found to be not proportional to the length scales of the quasi-ordered domains. They are instead related through a nontrivial power-law relation. The length scale of the quasi-ordered domains (as determined from optimal collapse of the correlation functions for the order parameter $\exp [3 i \theta(r)])$ does not follow a simple power law growth but exhibits an anomalous growth with time-dependent effective growth exponent. The breakdown of the critical dynamic scaling is accompanied by unusual relaxation dynamics in the decay of fractional $(3 \theta)$ vortices, where the decay of the vortex numbers is characterized by an exponential function of logarithmic powers in time.

PACS numbers: 64.70.qj, 64.60.Ht, 75.10.Hk
\end{abstract}

November 8, 2018

\section{INTRODUCTION}

Thermodynamic systems quenched from a hightemperature disordered phase into a low-temperature ordered phase exhibit characteristic growth of the length scale $\ell$ of the ordered domains which in typical situations can be represented as a power law $\ell \sim t^{1 / z}$, where the growth exponent $1 / z$ depends on the dimension of the space and of the relevant order parameter as well as on the conserved or nonconserved nature of the order parameter $\stackrel{1}{\underline{1}}$

Phase ordering dynamics (or coarsening process) is usually accompanied by the annihilation and decay of the characteristic topological defects such as point vortices or domain walls which are generated in the initial disordered states. One of the most important notions in understanding and analyzing these coarsening processes is the so-called dynamic scaling hypothesis ${ }^{\underline{1}}$ for the equaltime spatial correlation function of the order parameter, which is closely related to the observed self-similarity of the coarsening systems at different time instants.

When the low temperature phase of the system is characterized by a quasi-long-range order with power law decay of the spatial correlation function of the order parameter at equilibrium, then the dynamic scaling is generalized to the critical dynamic scaling. One of the well known examples is the ferromagnetic $X Y$ model $^{2,3}$ on the square lattice.

However, it should be noted that this (critical) dynamic scaling hypothesis for the phase ordering (or quasiordering) dynamics has not been proved on some general theoretical basis. Therefore, it is not clearly known what is the condition for the validity of the dynamic scaling which is usually assumed in analyses of experimental results or numerical simulations on coarsening dynamics.
In typical situations, coarsening dynamics is investigated on systems where the phase ordering is accompanied by the breaking of discrete or continuous global symmetry. However, there exist model systems that exhibit infinite ground state degeneracies of a discrete nature in addition to the usual (global) continuous symmetry. Prominent examples are geometrically frustrated spin systems such as antiferromagnetic Ising models on a triangular lattice ${ }^{4,5}$, antiferromagnetic $X Y$ or Heisenberg models on kagome $e^{\underline{6}}$ or pyrochlore lattices ${ }^{\underline{7}}$ These systems exhibit interesting equilibrium and nonequilibrium behavior due to the geometric frustration effect, including spin-glass-like relaxation dynamics without quenched disorder.

In this work we perform numerical simulations on the coarsening of an antiferromagnetic $X Y$ model on the kagome lattice (KAFXY model) $)^{8,9,10,11,12}$ which is one of the simplest geometrically frustrated models with infinite ground state degeneracies. Experimentally, this model can be realized in superconducting Josephsonjunction arrays or superconducting wire networks $\frac{13,14,15}{1}$ on a kagome lattice when a perpendicular magnetic field of half flux quantum (per plaquette) is applied on the system. We can also find physical examples in the anisotropic limit of Heisenberg antiferromagnets on the kagome lattice. It is well known that the system exhibits an infinite ground state degeneracy with finite entropy $\stackrel{16}{ }$. The system also exhibits a finite temperature $K T$ transition ${ }^{\underline{3}}$ corresponding to the unbinding of so-called fractional $3 \theta$-vortices. That is, at low temperature below the $K T$ transition, equilibrium of the system will be characterized by quasi-long-range order of the order parameter $\psi_{3} \equiv e^{3 i \theta(r)}$. Analogous to the case of simple ferromagnetic $X Y$ model on a square lattice, we might expect that the coarsening dynamics of the system would exhibit a critical dynamic scaling for the equaltime spatial correlation of the order parameter.

Our simulations, however, show that the critical dy- 
namic scaling is not obeyed very well at least for the time duration of our numerical simulations ranging several decades of time scale. That is, it was not possible to achieve a good scaling collapse for the equal-time spatial correlation functions of the $3 \theta$ order parameter. Another signature of the breakdown of the critical dynamic scaling is that the length scales corresponding to the average separation between fractional vortices are not proportional to the length scales of the growing quasi-ordered domains, which are instead related through a nontrivial power-law relation. This means that the two length scales exhibit different growth behavior in time. In terms of the decay of the fractional vortices, we also found that the fractional $3 \theta$-vortices residing in the small triangular plaquettes exhibit faster decay, while, in contrast, those vortices sitting on the larger hexagonal plaquettes exhibit much slower decay that can be fitted by an exponential of logarithmic powers in time ${ }^{17}$.

In addition to these features of multiple length scales in the coarsening dynamics, the time dependence of the length scale of the quasi-ordered domains does not exhibit a simple power law growth but rather exhibits an anomalous growth with time-dependent effective growth exponent. This appears to be closely related to the unusual relaxation dynamics in the non-power-law decay of the fractional $(3 \theta)$ vortices. It is not clear yet whether the scaling may be restored in the asymptotic limit.

\section{THE MODEL SYSTEM AND SIMULATION METHODS}

The kagome lattice consists of corner-sharing triangles (Fig. 11). In an antiferromagnetic $X Y$ model on the kagome lattice, the Hamilitonian is defined as

$$
H=-J \sum_{\langle i, j\rangle} \cos \left(\theta_{i}-\theta_{j}\right) .
$$

where $J<0$, the sum runs over all nearest neighbor pairs of sites, and $\theta_{i}$ denotes the angle of the planar spin at site $i$ with respect to some fixed direction in the two dimensional spin space. $\langle i, j\rangle$ indicates all pairs of nearest neighbor sites in the kagome lattice.

It is easy to see that the ground states of this system have the property that for all pairs of nearest neighbors $i$ and $j$, the angle difference satisfies $\left|\theta_{i}-\theta_{j}\right|=2 \pi / 3$ which means that the sum of the three spins vectors on any unit triangle vanishes. Thus the space of the ground states of KAFXY model are equivalent (up to a global rotation) to the ground states of the three state Potts model on the kagome lattice. In Figs. 1(a)-(b) are shown two examples of simple ground states with long-range order, the so-called $\mathbf{q}=0$ state and $\mathbf{q}=\sqrt{3} \times \sqrt{3}$ state, where $\mathbf{q}$ refers to the wave vector corresponding to the periodicity of the chirality configuration. It is easy to see, however, that in addition to these ground states with simple spatial order there also exist infinitely many ground states with no spatial order. It is well known that the system has a ground state entropy of $S_{0} \simeq 0.126 k_{B}$ per site $\frac{16}{}$. Now, if we consider the angle variable $3 \theta$ and the corresponding complex order parameter $\Psi_{3} \equiv \exp (i 3 \theta)$, the degenerate ground states are all completely ordered in terms of this new order parameter. And it has been shown that the system undergoes a $K T$ transition at a finite temperature, where the spatial correlation of the order parameter exhibits an algebraic decay below the transition temperature.

As for the $K T$ transition temperature of this system, an analytic approximation was given as $\frac{10,11}{1}$

$$
T_{c}=\frac{\pi \sqrt{3}}{72} J \approx 0.0756 J
$$

Numerical simulations were performed by Rzchowski ${ }^{9}$ where he found two slightly different estimates on the transition temperature, i.e., one estimate of $T_{c} \simeq 0.076 \mathrm{~J}$ based on the Binder cumulants of the order parameter and another of $T_{c} \simeq 0.070 \mathrm{~J}$ from the helicity modulus (or correspondingly the decay exponent $\eta\left(T_{c}\right)=0.25$ of the spatial correlation of the order parameter).

In the present work, the coarsening dynamics of the model system is performed via kinetic Monte Carlo methods with standard Metropolis algorithm for quenches to various temperatures near and below the $K T$ transition. System sizes ranging up to $N \times N=256 \times 256$ were employed with periodic boundary conditions. In a kagome lattice, the number $\widetilde{N}$ of the total spins is $\widetilde{N}=\frac{3}{4} N^{2}$. The system is quenched from completely disordered initial states down to a given low temperature with the process of coarsening being monitored through equal-time spatial correlation functions and the decay of topological defects, etc.

In addition to quenches from disordered state to low temperatures, we also performed the so-called nonequilibrium relaxation by suddenly bringing the system from one of the ground states to some target temperatures around or below the $K T$ transition. This method was found to be convenient for measuring the values of the critical exponent $\eta$ for the equilibrium spatial correlation functions.

When we let the systems evolve from random initial configurations the following quantities can be measured:

1. The number density $n_{v}(t)$ of topological defects which are $3 \theta$-vortices in the KAFXY model

$$
n_{v}(t) \equiv \frac{\left\langle N_{v}(t)\right\rangle}{\tilde{N}},
$$

where $N_{v}(t)$ is the total number of $3 \theta$-vortices (both positive and negative) at time $t$ and $\tilde{N}$ denotes the total number of sites (i.e., spins). $\langle\cdots\rangle$ denotes an average over random initial configurations. We also count the separate number density of vortices residing on the hexagonal plaquettes $\left(n_{h}\right)$ and those on the triangular plaquettes $\left(n_{t}\right)$. 
2. The equal-time spatial correlation function of the $3 \theta$ order parameter $\psi_{3}(\mathbf{r}, t) \equiv e^{3 i \theta(\mathbf{r}, t)}$.

$$
\begin{aligned}
C(r, t) & =\left\langle\psi_{3}^{*}(\mathbf{r}, t) \psi_{3}(0, t)\right\rangle \\
& =\frac{1}{\widetilde{N}}\left\langle\sum_{i} \exp \left(3 i \theta_{i}(t)-3 \theta_{i+r}(t)\right)\right\rangle
\end{aligned}
$$

3. Nonequilibrium spin autocorrelation functions

$$
\begin{aligned}
A(t) & =\frac{1}{\widetilde{N}}\left\langle\sum_{i} \exp \left(i \theta_{i}(0)-\theta_{i}(t)\right)\right\rangle \\
A_{3}(t) & =\frac{1}{\widetilde{N}}\left\langle\sum_{i} \exp \left(3 i \theta_{i}(0)-3 \theta_{i}(t)\right)\right\rangle .
\end{aligned}
$$

\section{SIMULATION RESULTS}

We have performed dynamic Monte Carlo simulations of $K A F X Y$ model on a kagome lattice of dimensions $256 \times 256$. In order to obtain the values of the equilibrium critical exponents $\eta$ for the spatial decay of the equilibrium spatial correlation function $C_{e q}(r)$ of the $3 \theta$ order parameter, we have employed the so-called nonequilibrium relaxation (NER) method, where the system is suddenly brought from ground states to finite temperatures below or near the $K T$ transition. Simulations were performed up to 655360 Monte Carlo steps which was sufficient for the equilibrium to be attained. This was confirmed by the collapse of the spatial correlations at later time stages. In Fig. 2(a) the correlation functions $C_{e q}(r)$ are displayed on a $\log -\log$ scale. The values of the exponent $\eta$ thus determined for temperatures ranging from $T=0.01$ to $T=0.074$ are shown in Fig. 2(b) as well as in Table [

\begin{tabular}{|c|c||c|c|}
\hline \hline$T / J$ & $\eta(T)$ & $T / J$ & $\eta(T)$ \\
\hline 0.01 & $0.047(3)$ & 0.065 & $0.254(11)$ \\
\hline 0.02 & $0.082(4)$ & 0.066 & $0.259(13)$ \\
\hline 0.03 & $0.116(5)$ & 0.067 & $0.264(12)$ \\
\hline 0.04 & $0.153(8)$ & 0.068 & $0.268(12)$ \\
\hline 0.05 & $0.198(7)$ & 0.069 & $0.280(11)$ \\
\hline 0.06 & $0.235(9)$ & 0.070 & $0.300(13)$ \\
\hline 0.061 & $0.237(10)$ & 0.071 & $0.308(12)$ \\
\hline 0.062 & $0.240(11)$ & 0.072 & $0.310(13)$ \\
\hline 0.063 & $0.245(10)$ & 0.073 & $0.330(15)$ \\
\hline 0.064 & $0.250(12)$ & 0.074 & $0.340(13)$ \\
\hline \hline
\end{tabular}

TABLE I: The equilibrium exponent $\eta$ for different temperatures for the power-law decay of the spatial correlation of the $3 \theta$ order parameter.

As for the generation of the initial low energy configurations, we first select one of the three phases of 0 , $\pm 2 \pi / 3$ for each site at random and then applied Monte Carlo procedure at very low temperature of $T=0.005$ for $10000 \mathrm{MC}$ steps with the restriction that, in each of the MC procedure, a new trial phase is chosen only among the above three phases. In this way, the final states obtained were very close (in energy) to a ground state. These states were employed as the initial states of $N E R$ in order to bring the system to the equilibrium at some given target temperature.

From the graph of $\eta(T)$ shown in Fig. 2(b), we find that the exponent increases almost linearly in temperature up to around $T \simeq 0.065$, above which it starts to increase rather sharply. The linear regime can be fit approximately by $\eta(T) \simeq 3.9 T$. We also find that the exponent $\eta$ takes the value of $1 / 4$ near $T(\eta=1 / 4) \simeq 0.064 J$ (Table 【). This temperature is expected to correspond to the $K T$ transition, which apparently is a little lower than the theoretical ${ }^{10,11}$ or the numerical ${ }^{9}$ estimates of earlier works mentioned above.

Now, the coarsening dynamics of the model system under quench to low temperature from disordered initial states is investigated by monitoring the equal-time spatial correlation functions of the $\psi_{3}$ order parameter. One of the most important features of typical coarsening dynamics toward quasi-ordered phase is the critical dynamic scaling

$$
C(r, t)=r^{-\eta(T)} f(r / L(t)),
$$

where $f(x)$ is the scaling function and $L(t)$ is the growing length scale. It should be noted that the above scaling ansatz is based on the existence of a single growing length scale $L(t)$.

Figure 3(a)-(b) shows the equal-time correlation functions $C(r, t)$ of $K A F X Y$ model for different time instants (from $t=10$ to 655360 ) at the temperature $T=0.060$. For these data, we attempted a critical dynamic scaling. The procedure of rescaling is as follows. For a given temperature, we take the value of $\eta(T)$ (determined as in Table I) and evaluate the combination $\widetilde{C}(r, t) \equiv r^{\eta(T)} C(r, t)$. And then, for a given time instant, the length scale $L(t)$ is determined by the condition $\widetilde{C}(r=L(t), t)=C_{0}$ where the constant $C_{0}$ is chosen as $C_{0}=0.2$ or $C_{0}=0.3$ in this work. By plotting the scaled correlation functions $\widetilde{C}(r, t)$ in terms of the rescaled distance $r / L(t)$, we can check whether the critical dynamic scaling holds or not from the quality of the collapse of the scaled correlation functions.

By this procedure, we found, rather unexpectedly, that the critical dynamic scaling does not hold in the coarsening dynamics of KAFXY model, at least for the (Monte Carlo) time duration of our simulations (up to 655360 MC steps). The result of the scaling attempt is shown in Fig. 3(c) for the case of $T=0.06$ where we can see that the critical dyanmic scaling is not obeyed. We also tried different values of $\eta$ for the rescaled correlation with no success. It might be possible that the scaling is restored in the limit of infinitely long time. 
Even though the critical dynamic scaling is not faithfully obeyed, we may still extract approximate length scale $L(t)$ of spatial correlation in the manner described above. The length scale $L(t)$ thus obtained exhibits a rather unusual time-dependent behavior (Fig. 3(d)). That is, in the early-time stage up to around $t \sim 10^{3}, L(t)$ exhibits a slow growth, which appears approximately independent of the temperature. However, in the intermediate and late time stage of $t \gtrsim 10^{3}$, the growth of the length scale $L(t)$ becomes strongly dependent on the temperature. In addition, for a given temperature, no simple power law is found which is valid for the whole late time regime. Instead the local logarithmic slope exhibits a steady increase in time in the late time regime.

We can investigate the time-dependence of the growth of the length scale by defining the effective local growth exponent as $\beta(t) \equiv d \ln (L(t) / d \ln (t)$. Since we took the growing length scale $L(t)$ only for discrete time instants $t_{i}$ with fixed interval in logarithmic scale, we evaluate the discrete version of the above logaritmic slope as

$$
\beta\left(t_{i}^{\prime}\right) \simeq \frac{\ln \left(L\left(t_{i+1}\right) / L\left(t_{i}\right)\right)}{\ln \left(t_{i+1} / t_{i}\right)}
$$

where $t_{i} \equiv 2^{i}, i=1,2, \cdots$ and $t_{i}^{\prime} \equiv \sqrt{t_{i} t_{i+1}}$. Figures. 3(e)-(f) show the effective growth exponent at time $t$ (for the case of $C_{0}=0.2$ (Fig. 3(e)) and $C_{0}=0.3$ (Fig. 3(f)) respectively). We can see that, in the late time regime, the growth becomes definitely faster as the temperature is higher. Also, for the temperatures $T \gtrsim 0.05$, the effective local growth exponent appears to be monotonically increasing in the late time stage. At higher temperatures, we can recognize some indications from Figs. [3(e)-(f) that the effective slopes tend to converge around $0.5 \sim 0.6$ in the latest time regime. It may be possible to interpret this as an indication of an asymptotic behavior of diffusive growth.

In order to understand the physical mechanism underlying the breakdown of the critical dynamic scaling as well as the peculiar time-dependence of the length scale, we investigated the time dependence of the total number of the relevant topological defects, i.e., the fractional $3 \theta$ vortices residing on the triangular plaquettes as well as on the hexagonal plaquettes of the kagome lattice. The results are shown in Figs. ??(a)-(d) where we can see that, for a given temperature, the triangular vortices are decaying much faster than the hexagonal vortices. We also find that in general (for both types of the vortices) the decay of the total vortex numbers do not exhibit a simple power law behavior valid for the whole time range. One prominent feature in terms of the temperature dependence of the decay of the fractional vortex density is that, for the vortices on the triangular plaquettes, the decay rate increases as the temperature is lowered, while on the other hand, those vortices on the hexagonal plaquettes exhibit opposite dependence on the temperature with the decay rate decreasing sharply as the temperature is lowered.
This can be interpreted as implying that there is almost no (free) energy barrier for the motion and decay of the triangular vortices but that some finite barrier exists for the hexagonal vortices. We also find an interesting feature of the hexagonal vortices (at lower temperatures) where, at initial stage, they increase a little and then start to decay. This can probably be understood as the influence of the decay of the neighboring triangular vortices with their excess energy turned over to neighboring hexagonal vortices, thus generating hexagonal vortices.

Therefore, we can conclude that, for wide range of temperatures, the relaxation of the fractional vortices residing on the hexagonal plaquettes determines the coarsening process such as the correlation length scale corresponding to the quasi-ordered domains. In addition, the vortex relaxations exhibit a considerable deviation from power law behavior with slowly increasing (in absolute magnitude) local logarithmic slope in the late time regime. Especially in the case of the hexagonal vortices, the decay of the vortex number density could be fit to a form with

$$
\begin{aligned}
N_{h} & =N_{0} \exp \left[-b(\ln (t))^{\alpha}\right] \\
& =N_{0} \exp \left[-\left(\frac{\ln (t)}{\ln \left(t_{0}\right)}\right)^{\alpha}\right] .
\end{aligned}
$$

with $b \equiv \ln \left(t_{0}\right)^{-\alpha}$. Here, we found that typically $\alpha$ takes values around $3.3 \sim 4.0$ (figure not shown here ${ }^{18}$ ). We found that it could also be fitted to an stretched exponential form as

$$
N_{h}(t) \sim N_{0} \exp \left[-\left(t / t_{0}\right)^{\alpha}\right]
$$

with $\alpha \simeq 0.226 \pm 0.03$.

It appears that the breaking of the critical dynamic scaling is related to the anomalous relaxation of the number of fractional vortices on the triangular and the hexagonal plaquettes. In order to check this possibility, we analyzed the behavior of the length scales determined by the vortex distributions as follows. We define the length scale $\xi_{t} \equiv 1 / \sqrt{n_{t}}$ which corresponds to the average separation between the vortices on the trianular plaquettes. Similarly, we can define the length scale $\xi_{h} \equiv 1 / \sqrt{n_{h}}$ corresponding to the average separation between vortices on the hexagonal plaquettes. We also define the length scales $\xi_{v} \equiv 1 / \sqrt{n_{v}}=1 / \sqrt{n_{h}+n_{t}}$ which represents the length scale corresponding to the total number density of vortices.

Figure 5 shows the three length scales at $T=0.06$ which clearly shows the faster growth of the length scale for the triangular vortices compared with that for the hexagonal vortices. In Fig. ??(a)-(d) comparison is made between the length scales $L(t)$ derived from the spatial correlation functions and the length scales $\xi_{v}$ derived from the total vortex densities. We see that (for both cases of $C_{0}=0.2$ and $C_{0}=0.3$ ) there exists no simple proportional relationship between the two length scales. Rather, the length scale $L(t)$ from the spatial correlation is seen to grow faster than the vortex lenth scale $\xi_{v}$. This 
probably implies that the vortices are not distributed evenly (statistically speaking) in the system but that the vortices are somehow distributed in a non-random manner such that some degree of clustering occurs. Interesting result is that the two length scales satisfy some nontrivial relationships such that $\xi_{v} \sim L(t)^{\lambda}$ with $\lambda \simeq 0.65$ for the case of $C_{0}=0.2$ (Fig. ??(b)) and $\lambda \simeq 0.68$ for the case of $C_{0}=0.3$ (Fig. ??(d)). This means that the vortex configuration of the system do not exhibit simple selfsimilarity at different time instants. Rather the vortices probably tend to cluster more unevenly as time passes by, leading to the correlation length scale $L(t)$ growing faster than the length scale derived from the vortex density.

The snapshot of defect configurations for the case of $T=0.06$ is shown in Fig. ??(a)-(d). We can easily recognize faster decay of those vortices on the triangular plaquettes. From the snapshots alone, however, it is not easy to detect the tendency of relative clustering of the vortices in the late time regime.

Now we turn to the autocorrelation functions of the variables $\exp (i \theta)$ and $\exp (3 i \theta)$. For these two variables, we find that the simulation results on the autocorrelations are very different. For the case of $\exp (i 3 \theta)$, we obtain a approximately power-law decay behavior $A_{3}(t) \sim t^{-\lambda}$ in the early time regime up to around $t \simeq 10^{3}$. The value of the exponent $\lambda$ ranges from $\lambda \simeq 0.56$ (for $T=0.01$ ) to $\lambda \simeq 0.90$ (for $T=0.07$ ). The exponent tends to increase slightly at higher temperatures. At later time, however, the autocorrelations decay faster than power-law relaxation of the early time stage. Due to the larger statistical fluctuations in the relaxation of the autocorrelation $A_{3}(t)$, we could not attempt a comparison of $A_{3}(t)$ with the growing length scale $L(t)$.

In contrast, in case of the phase $\exp (i \theta)$, the spin autocorrelaton exhibits less statistical fluctuations with a non-power law behavior $\frac{17}{17}$ that can be reasonably fitted by

$$
\begin{aligned}
A(t) & =A_{0} \exp \left[-b(\ln (t))^{\gamma}\right] \\
& =A_{0} \exp \left[-\left(\frac{\ln (t)}{\ln \left(t_{0}\right)}\right)^{\gamma}\right]
\end{aligned}
$$

with the exponent $\gamma=2.0 \pm 0.3$ and $b \equiv \ln \left(t_{0}\right)^{-\gamma}$. One of the simulation results is shown in Fig. ??(a-b) for the case of $T=0.06$ with $\widetilde{N}$, which shows a suitable fit to the above functional form with $b \simeq 0.15$ and $\gamma \simeq 2.0$. Note that, in the limit of $\gamma=1, A(t)$ reduces to a power law of $A(t) \sim A_{0} t^{-b}$. The fitted values of $\gamma$ implies that $A(t)$ exhibits a considerable deviation from a power-law behavior. We also attempted to fit the simulation results to stretched exponential form. This resulted in rather small values for the stretching exponent of $\alpha \simeq 0.14$. It is interesting to note that similar behavior of relaxation in the autocorrelation function of the order parameter was reported in the coarsening dynamics of the so-called Hamiltonian $X Y$ model on a square lattice $\underline{\underline{19}}$

In summary, we investigated the coarsening dynamics of the antiferromagnetic $X Y$ model on a kagome lattice. We found that the critical dynamic scaling is violated. Novel and unusual coarsening dynamics may be attributable to the existence of infinite ground state degeneracies leading to nontrivial decay behavior of the density of defects residing on the triangular and hexagonal plaquettes with nontrivial growth of multiple length scales. The competition between the critical thermal fluctuations of the equilibrium and the infinite ground state degeneracy probably gives rise to the nontrivial features of the relaxation dynamics. It may be possible to collapse the equal-time spatial correlation functions through a multiscaling scheme which we haven't tried yet. It would be also interesting to investigate the aging dynamics of this model system $\underline{20}$
1 A. J. Bray, Adv. Phys. 43, 357 (1994).

2 V. L. Berezinskii, Sov. Phys. JETP 34, 610 (1972).

3 J. M. Kosterlitz and D. Thouless, J. Phys. C 6, 1181 (1973); J. M. Kosterlitz, J. Phys. C 7, 1046 (1974).

4 E. Kim, B. Kim, and S. J. Lee, Phys. Rev. E 68, 066127 (2003) and references therein.

5 E. Kim, S. J. Lee, and B. Kim, Phys. Rev. E 75, 021106 (2007).

6 I. Syozi, Prog. Theor. Phys. 6, 306 (1951).

7 R. Moessner and A. P. Ramirez, PHYSICS TODAY, February 2006, page 24.

8 D. A. Huse and A. D. Rutenberg, Phys. Rev. B 45, 75367539 (1992).

9 M. S. Rzchowski, Phys. Rev. B 55, 11745-11750 (1997).

10 V. B. Cherepanov, I. V. Kolokolov, and E. V. Podivilov, JETP Letters 74, 596 (2001).

11 S. E. Korshunov, Phys. Rev. B 65, 054416 (2002).

12 M. R. Kolahchi and J. P. Straley, Phys. Rev. B 66, 144502 (2002).
13 M. J. Higgins, Yi Xiao, S. Bhattacharya, P. M. Chaikin, S. Sethuraman, R. Bojko and D. Spencer, Phys. Rev. B 61, R894-R897 (2000).

14 K. Park and D. A. Huse, Phys. Rev. B 64, 134522 (2001).

15 Y. Xiao, D. A. Huse, P. M. Chaikin, M. J. Higgins, S. Bhattacharya, D. Spencer, Phys. Rev. B 65, 214503 (2002).

16 R. J. Baxter, J. Math. Phys. 11, 784 (1970).

17 V. B. Cherepanov, arXiv:cond-mat/9407068 (1994).

18 S. Park and S. J. Lee, unpublished.

19 K.-J. Koo, W.-B. Baek, B. Kim, and S. J. Lee, Jour. Kor. Phys. Soc. 49, 1977 (2006).

20 A. S. Wills, V. Dupuis, E. Vincent, J. Hammann, and R. Calemczuk, Phys. Rev. B 62, R9264 (2000) 


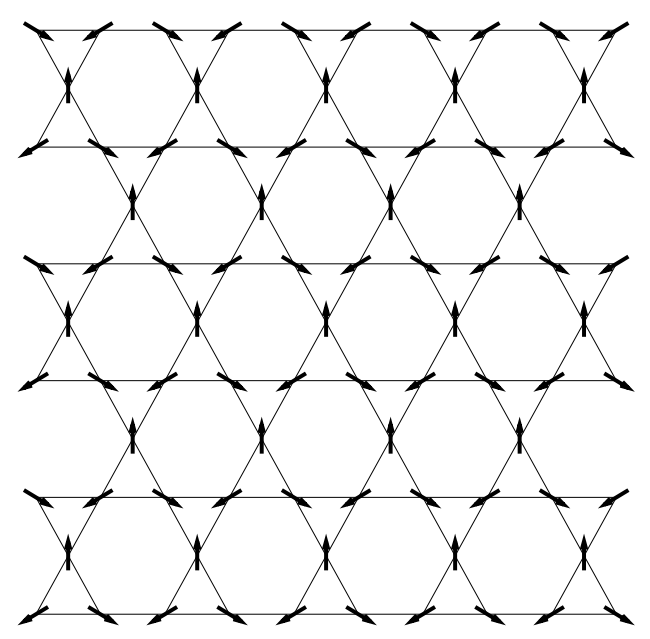

(a)

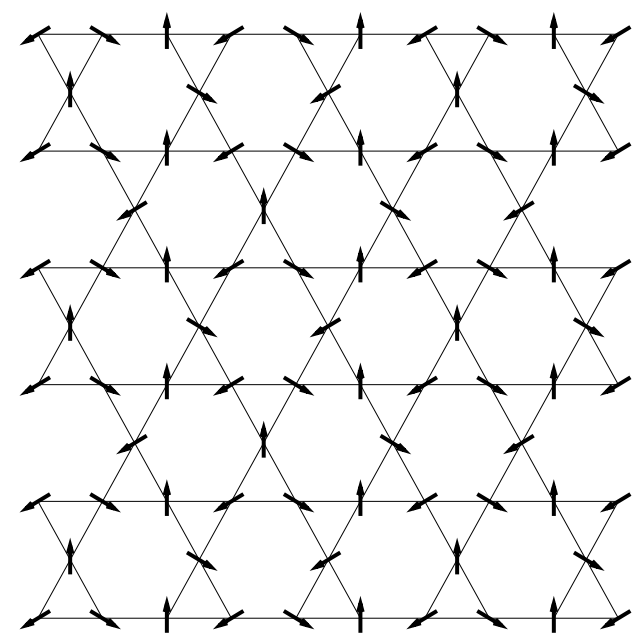

(b)

FIG. 1: Schematic drawings of kagome lattices with the so-called (a) $\mathbf{q}=0$ ground state and (b) $\mathbf{q}=\sqrt{3} \times \sqrt{3}$ ground state.
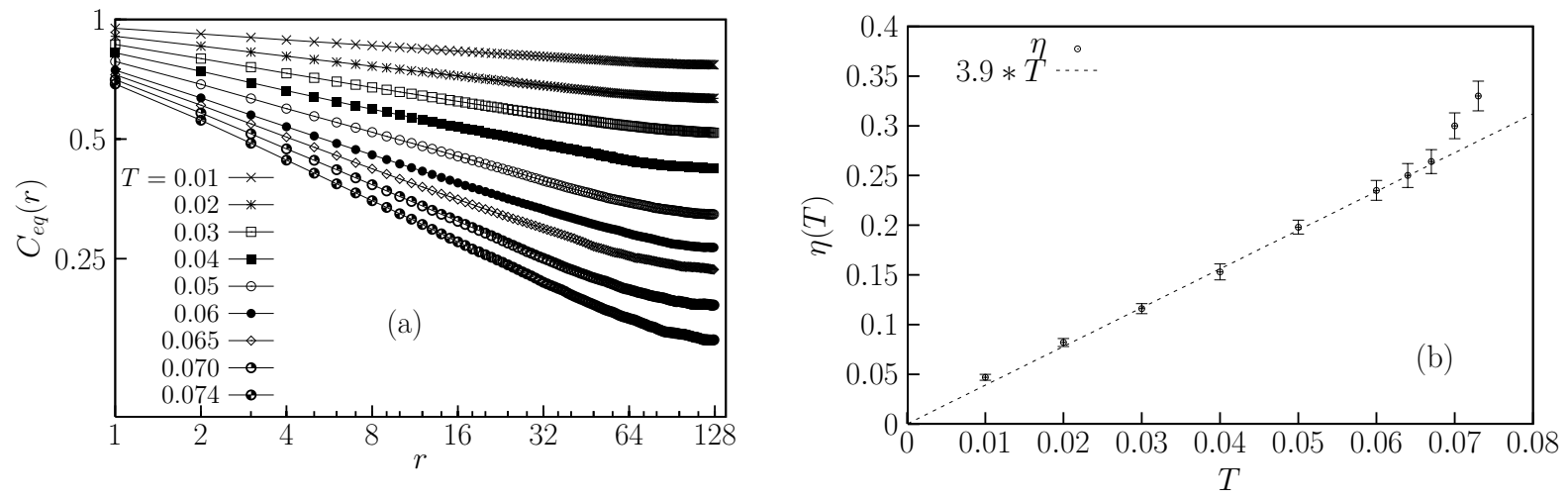

FIG. 2: (a) Equilibrium spatial correlation of $\exp (3 i \theta)$ at various temperatures and (b) the corresponding exponents $\eta(T)$ vs. $T$, obtained from nonequilibrium relxation method. In (b), the dotted line represent $3.9 T$ which fits reasonably well the behavior of $\eta(T)$ vs. $T$ especially at low and intermediate temperature regime $(T \leq 0.065)$. 

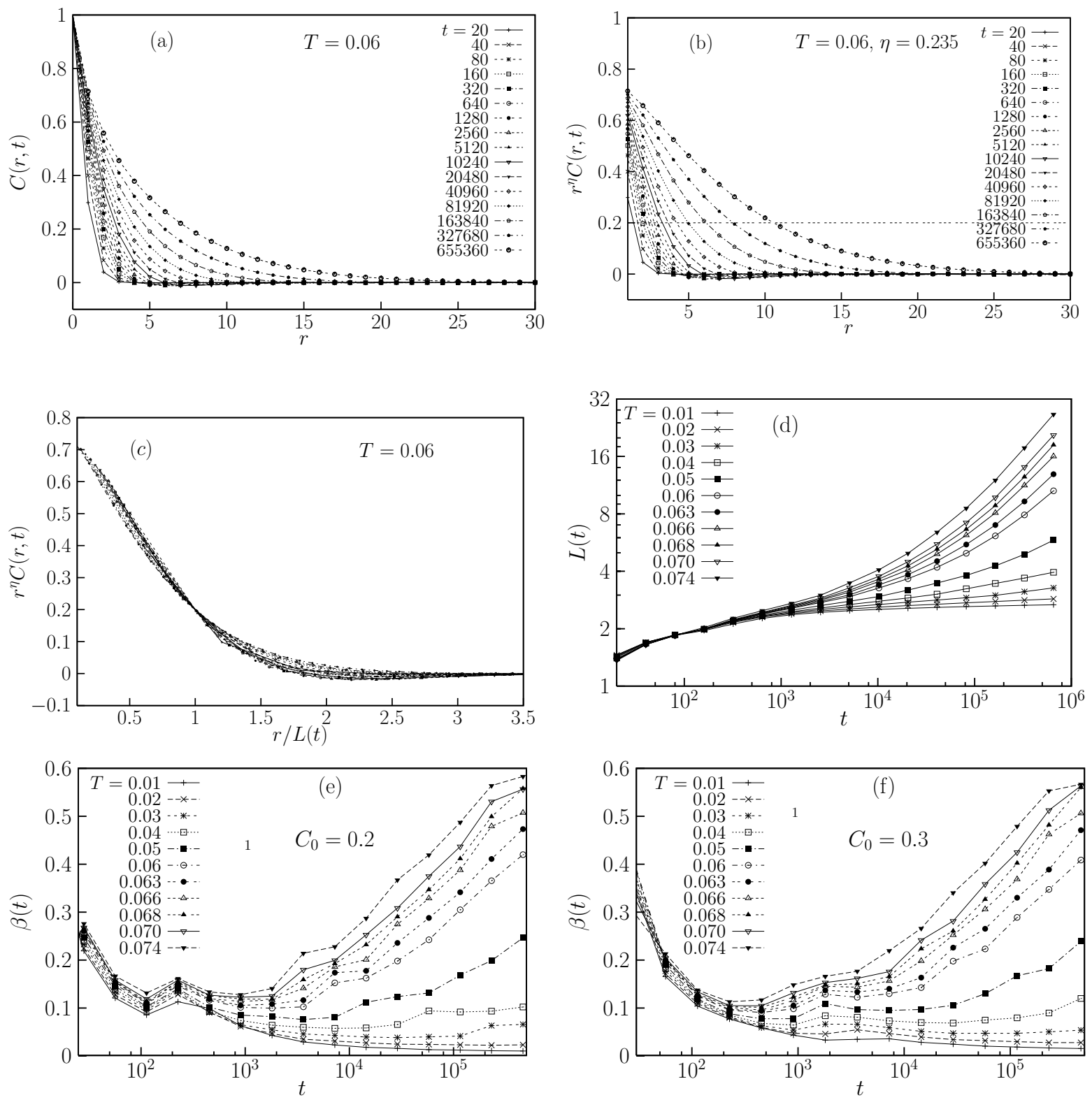

FIG. 3: (a) Equal-time spatial correlation function $C(r, t)$ for $\exp (3 i \theta)$ for different time instants at $T=0.06$, (b) the rescaled functions $\tilde{C}(r, t) \equiv r^{\eta}(T) C(r, t)$, (c) a scaling attempt based on (b) with $C_{0}=0.2$, (d) growth of the length scale (using $C_{0}=0.2$ ), (e) the effective growth exponents vs. time (using $C_{0}=0.2$ ), and (f) the effective growth exponents vs. time (with $\left.C_{0}=0.3\right)$. 


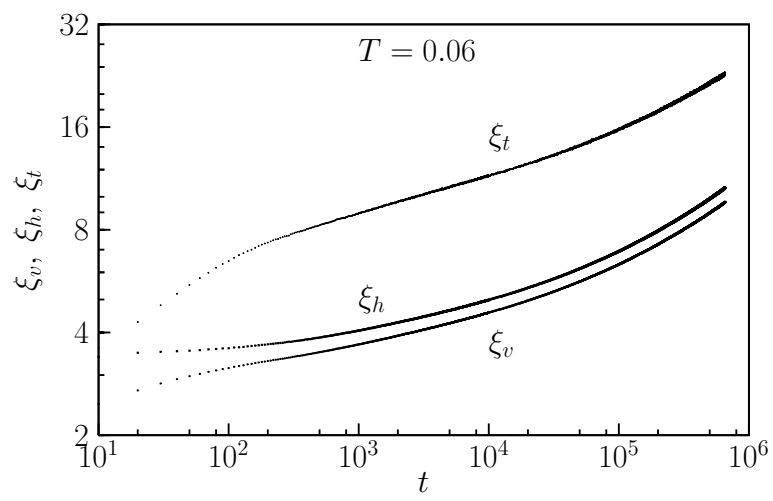

FIG. 5: The length scales $\xi_{h}, \xi_{t}$ and $\xi_{v}$ derived from the vortex densities versus time $t$ for hexagonal, triangular plaquettes and the sum of these, respectively at $T=0.06$. 


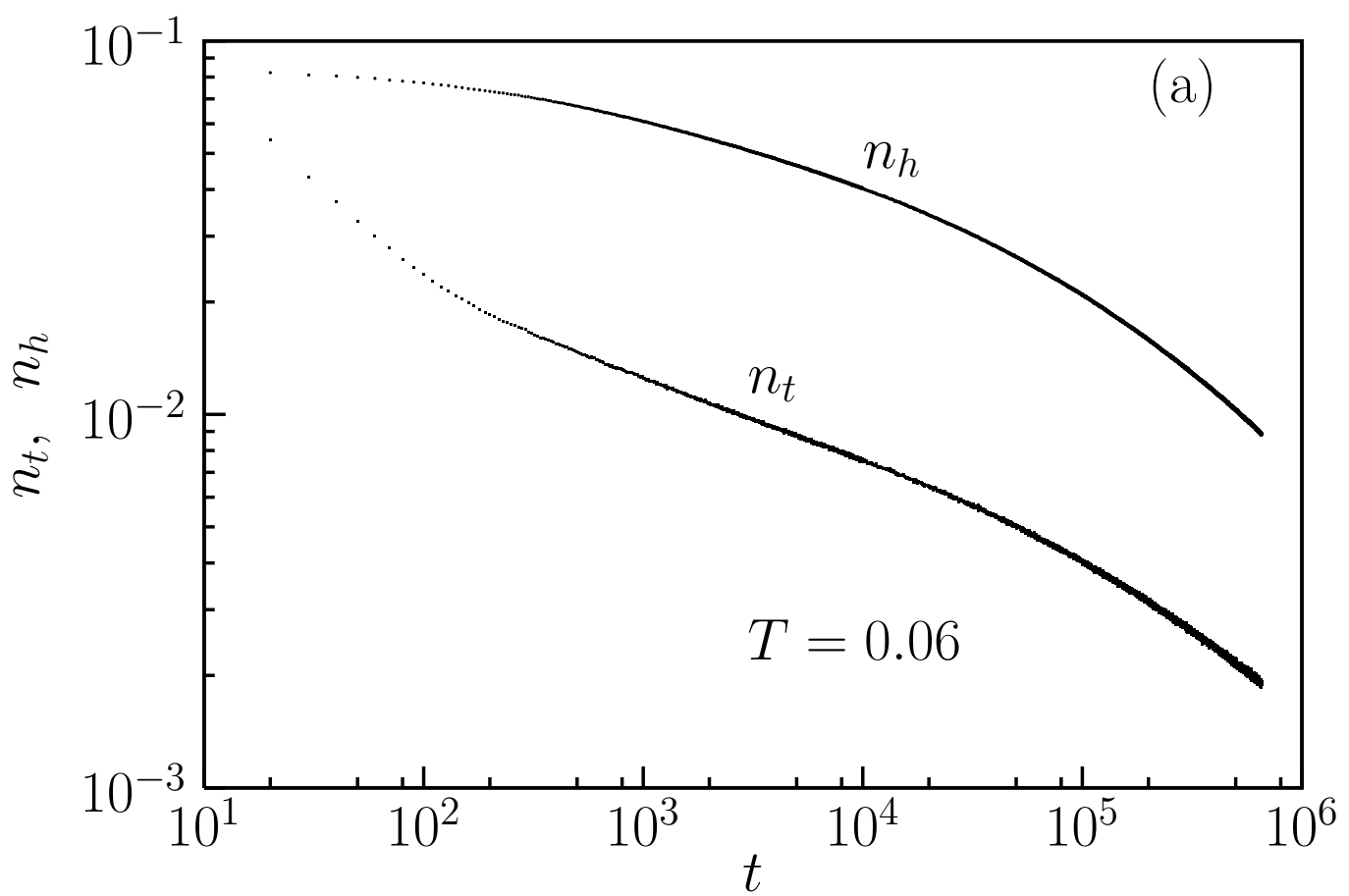




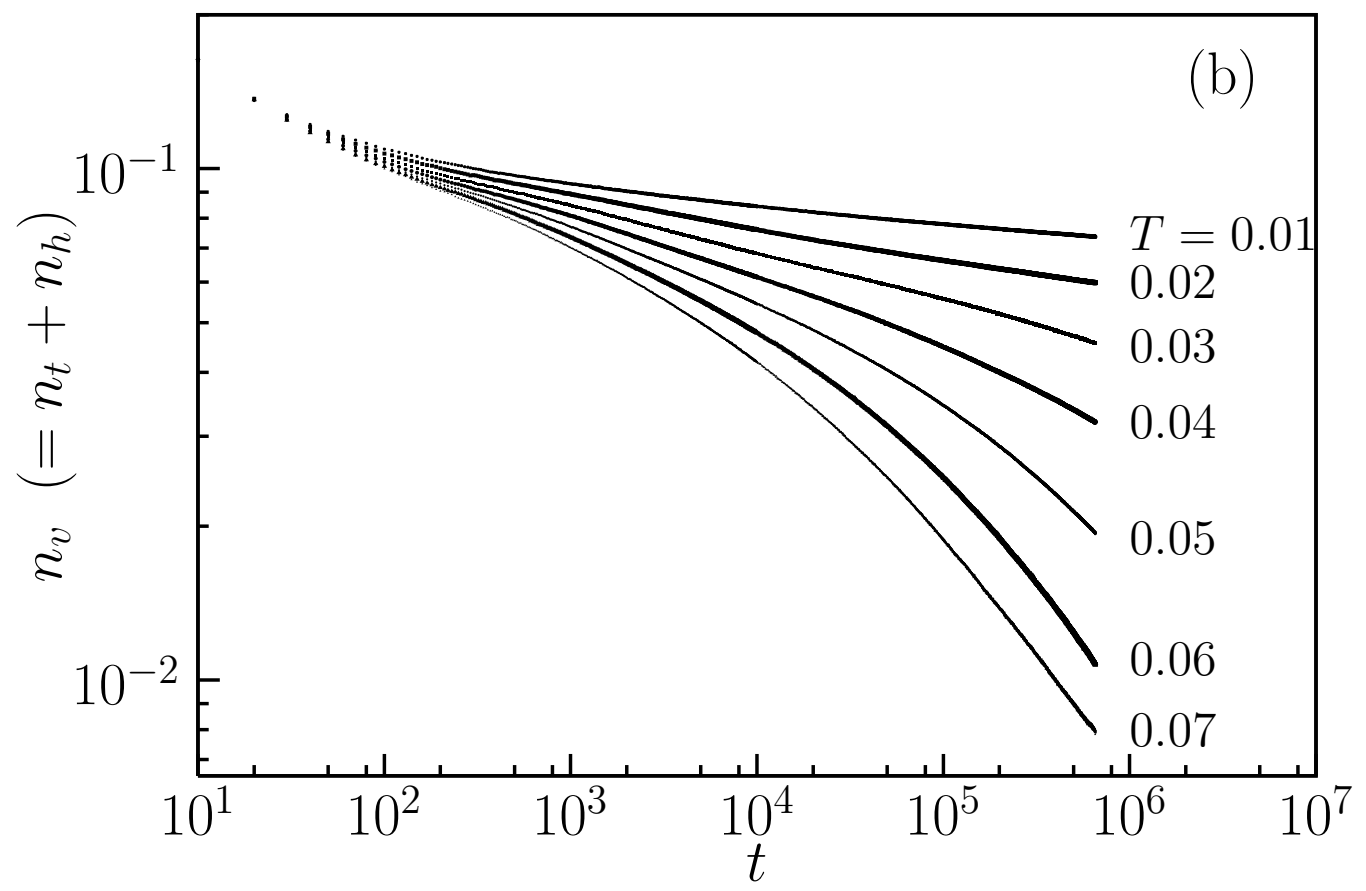




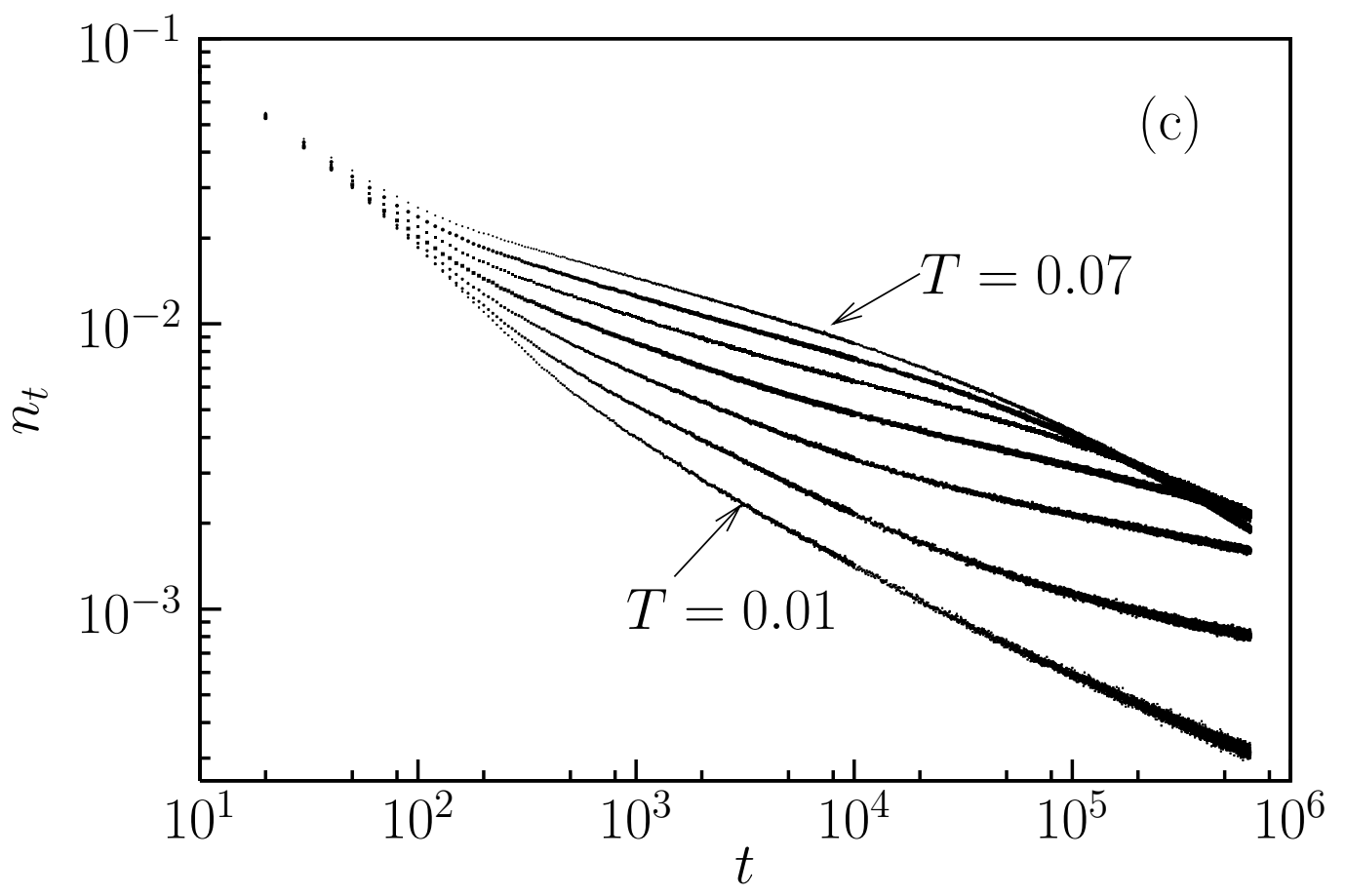




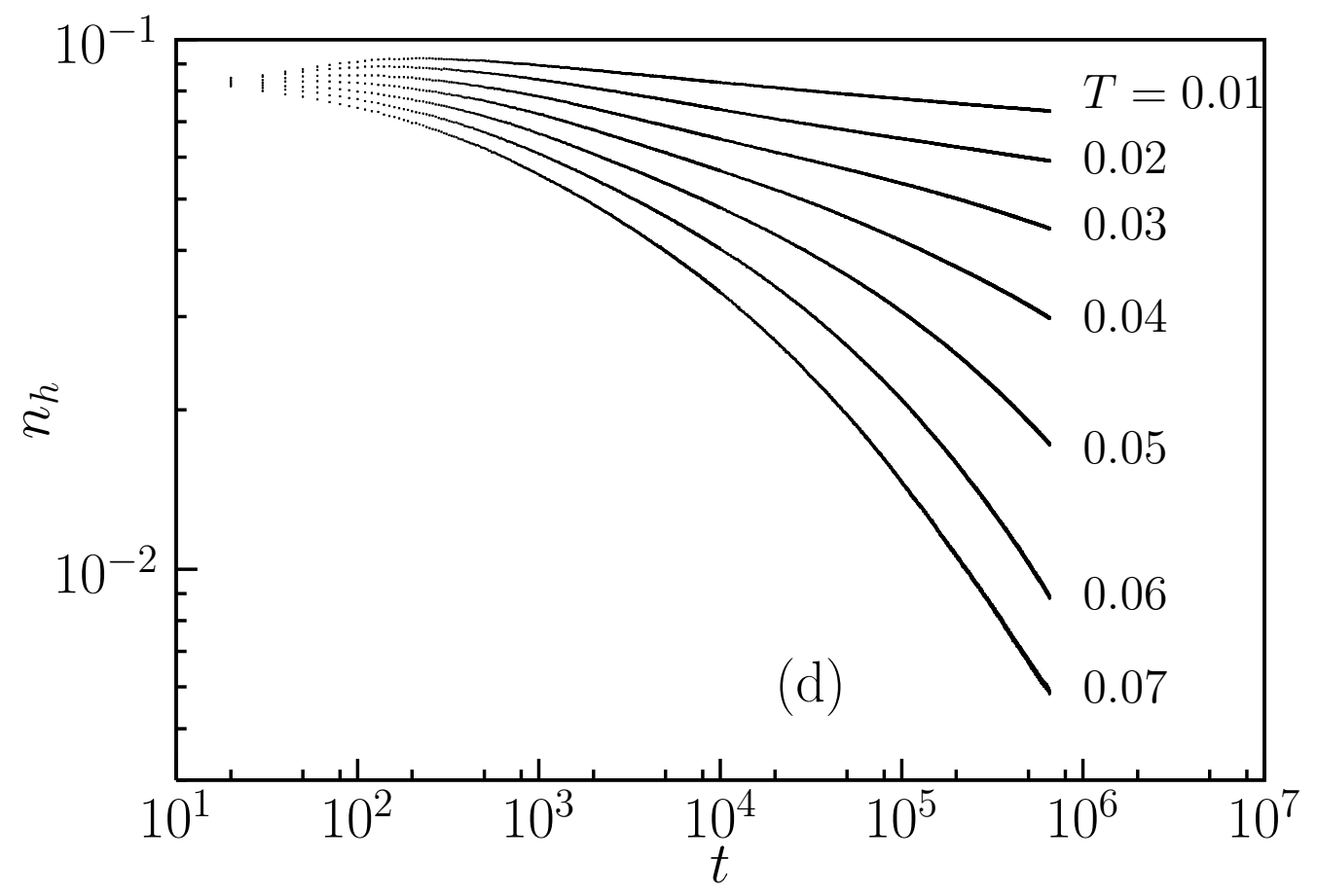




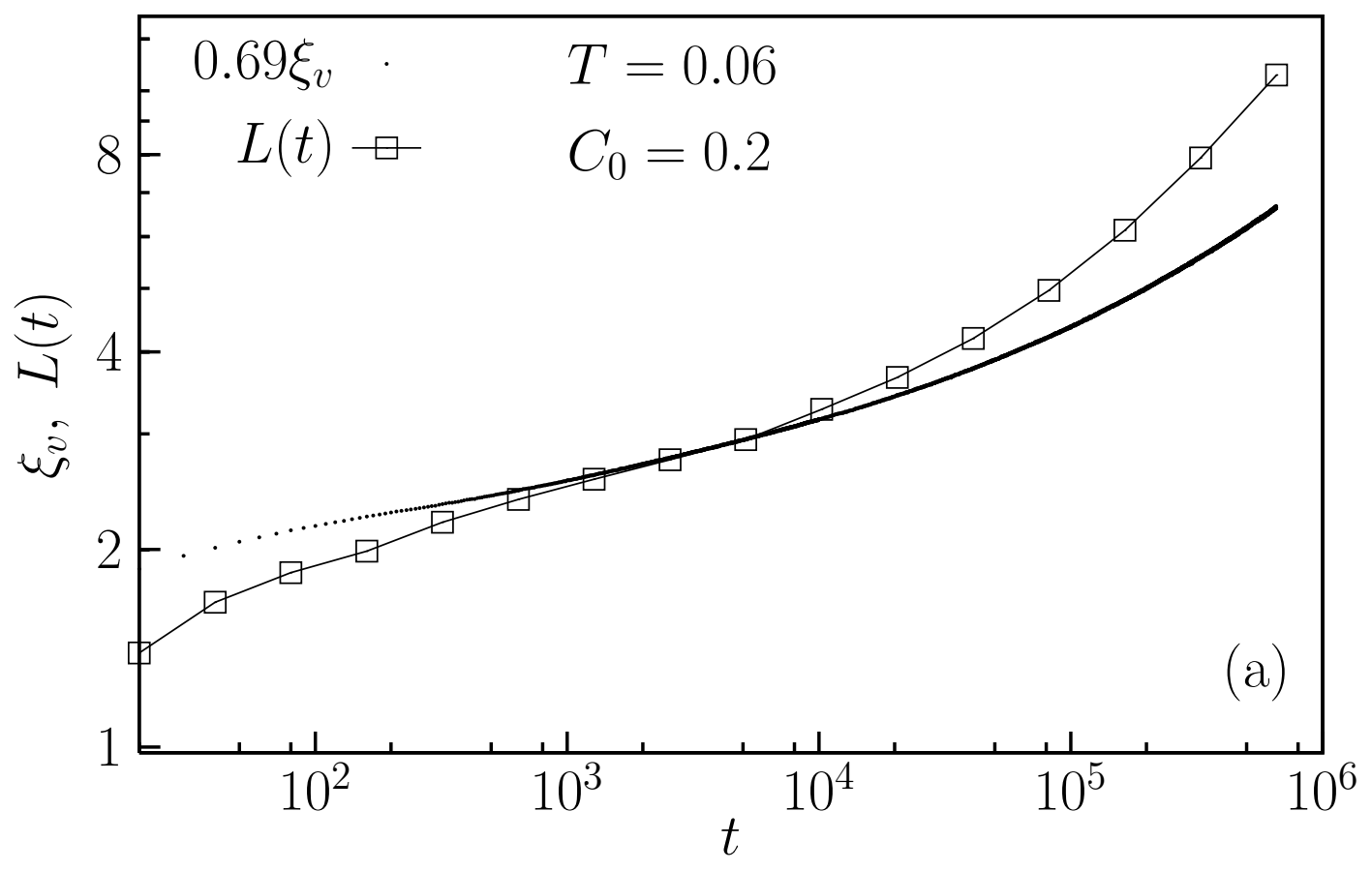




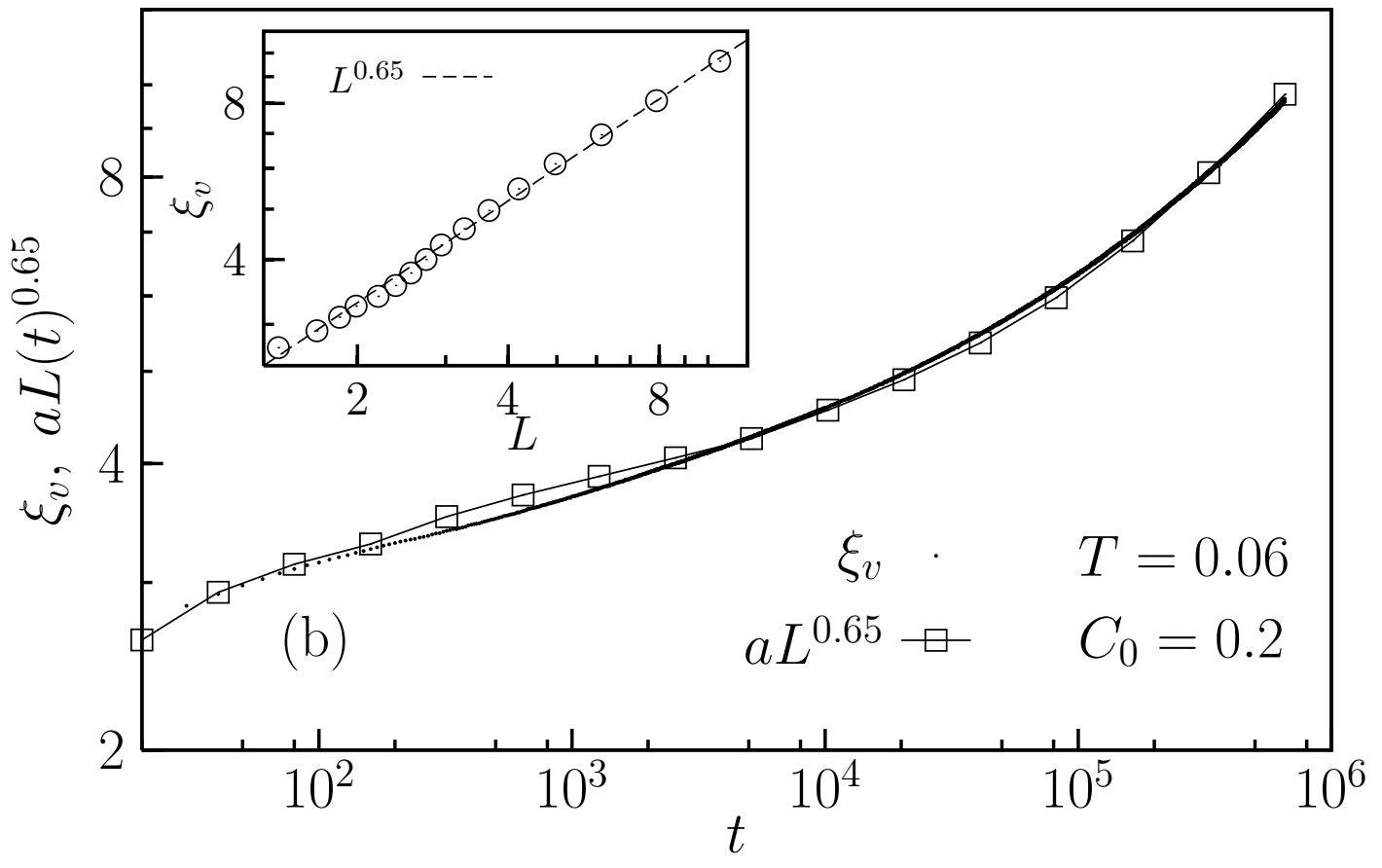




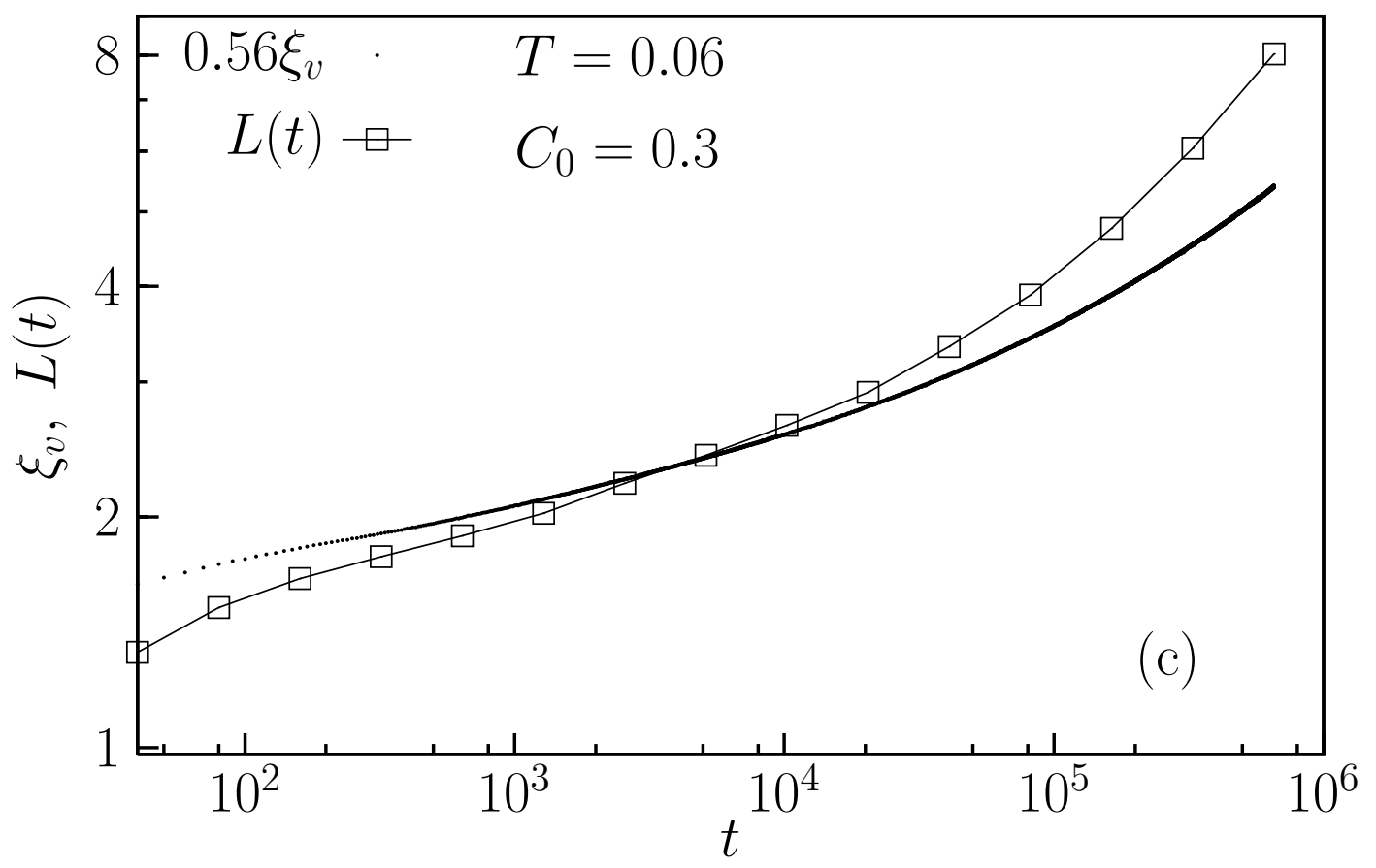




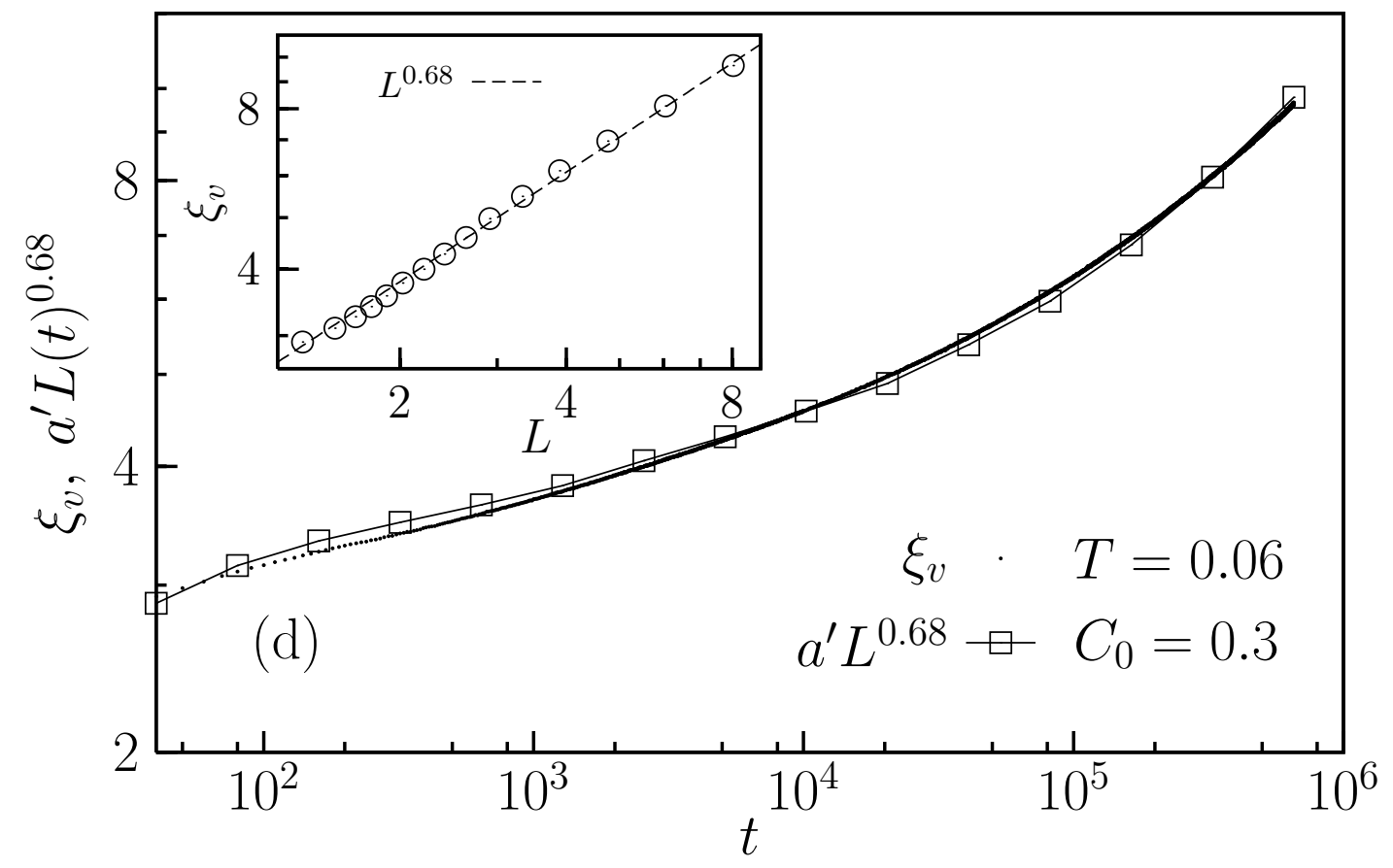




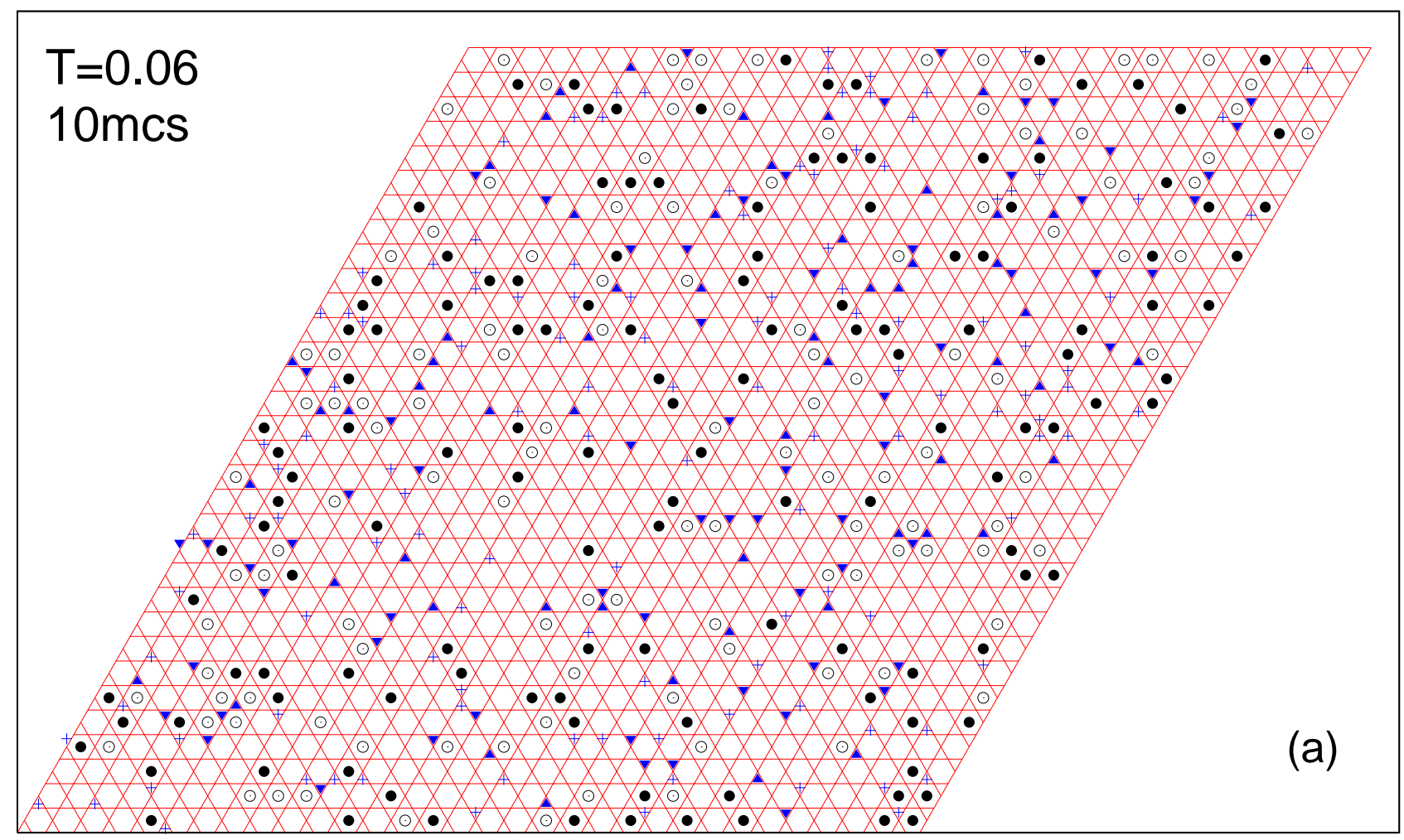




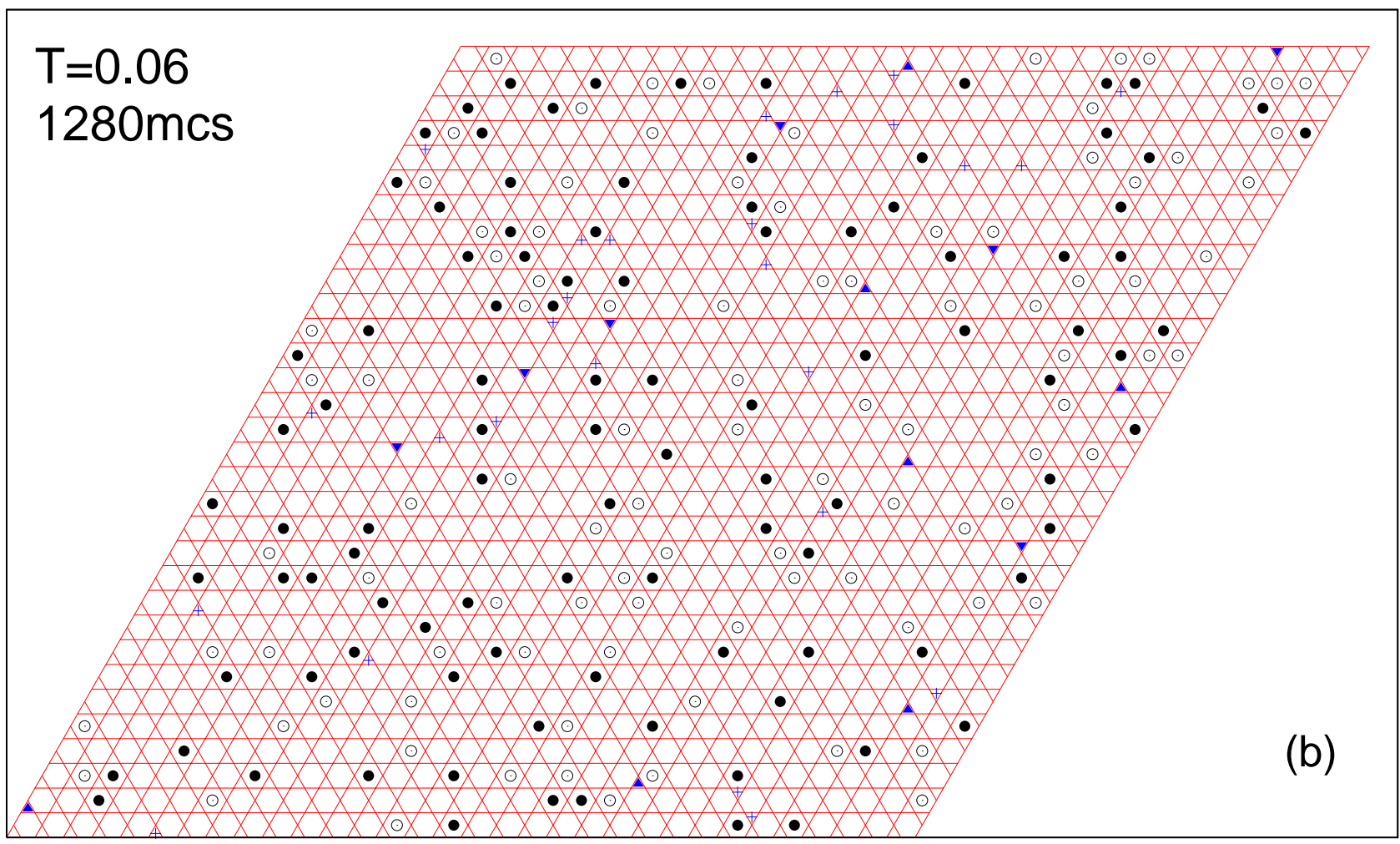




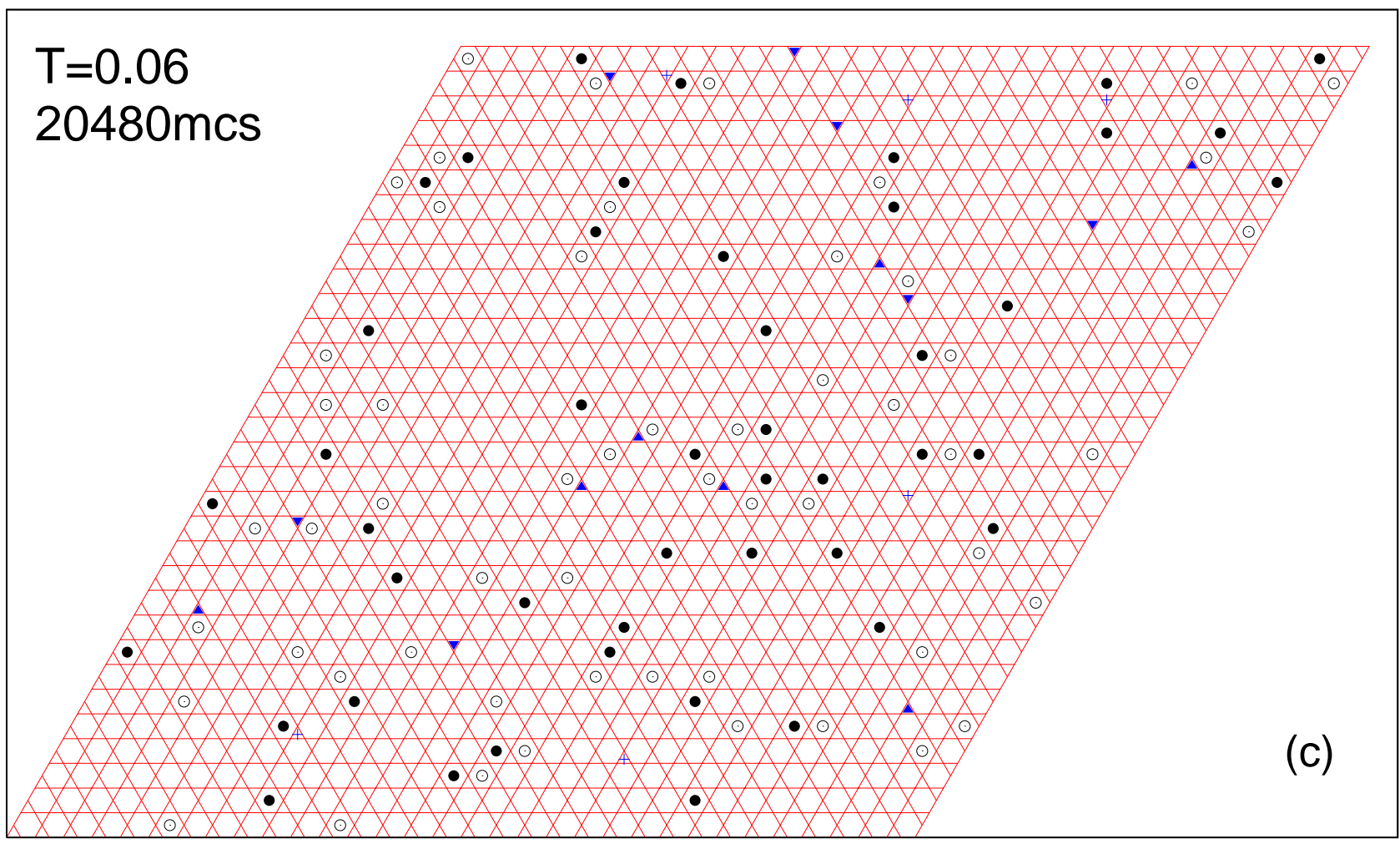




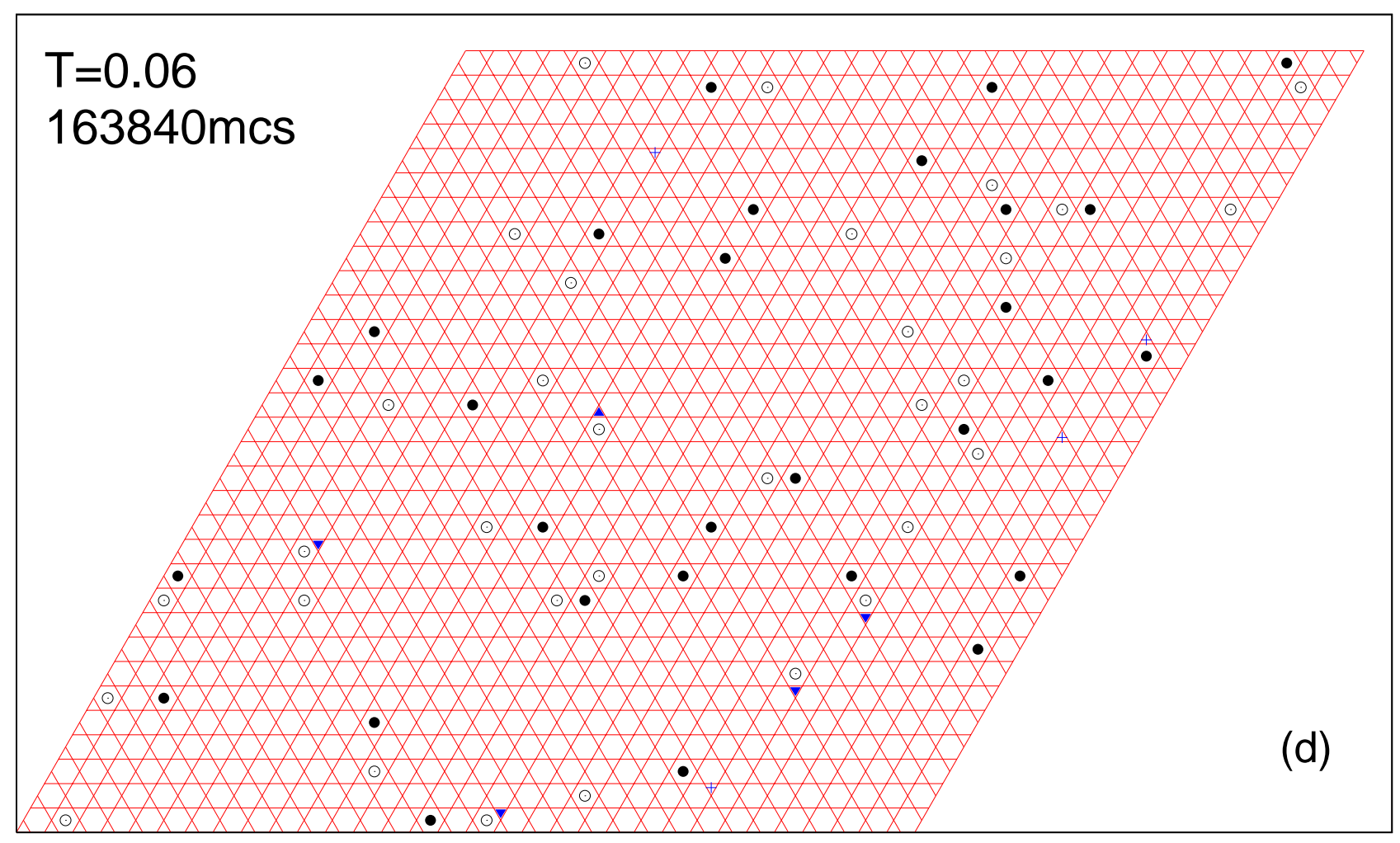




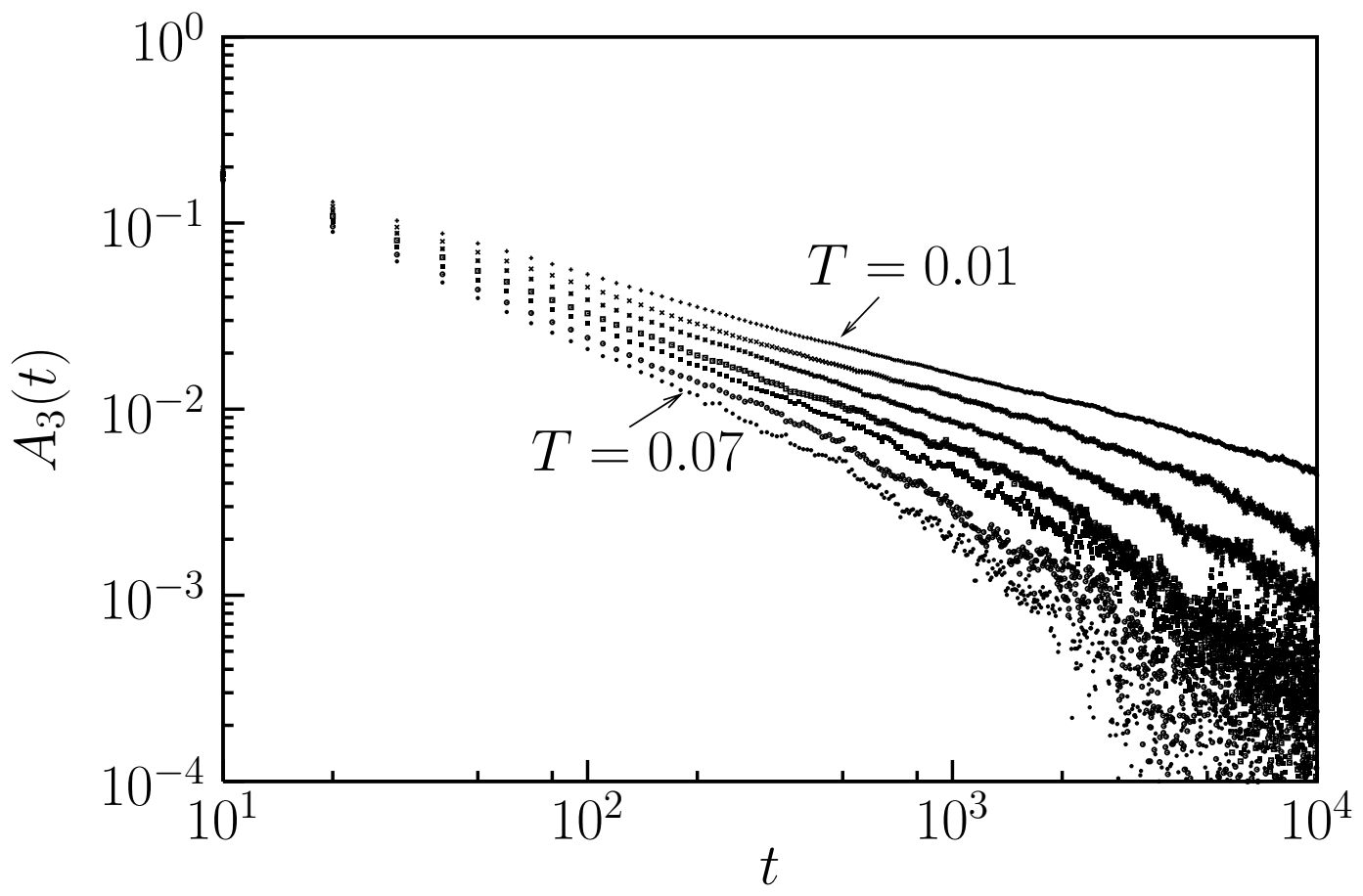




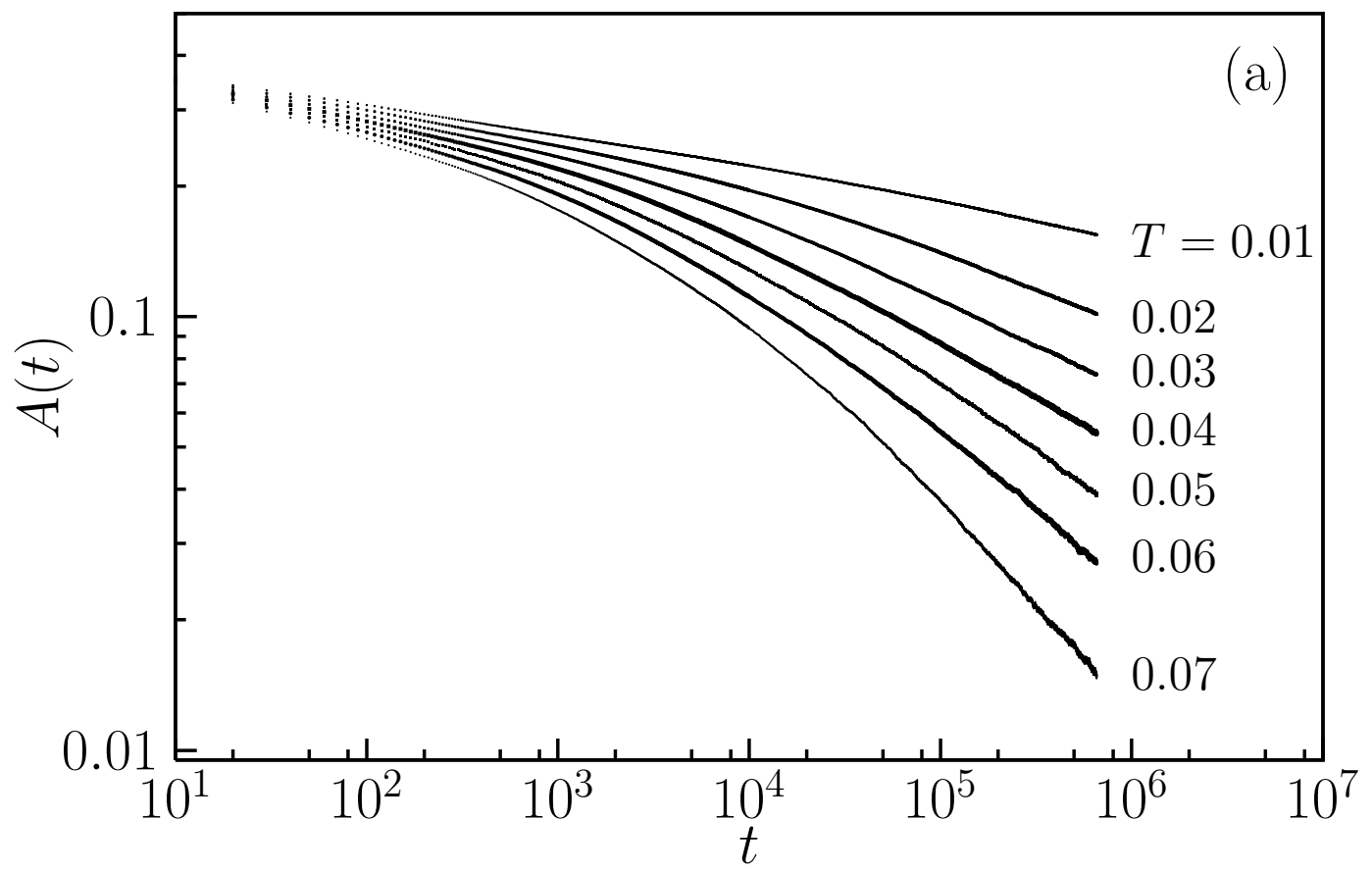




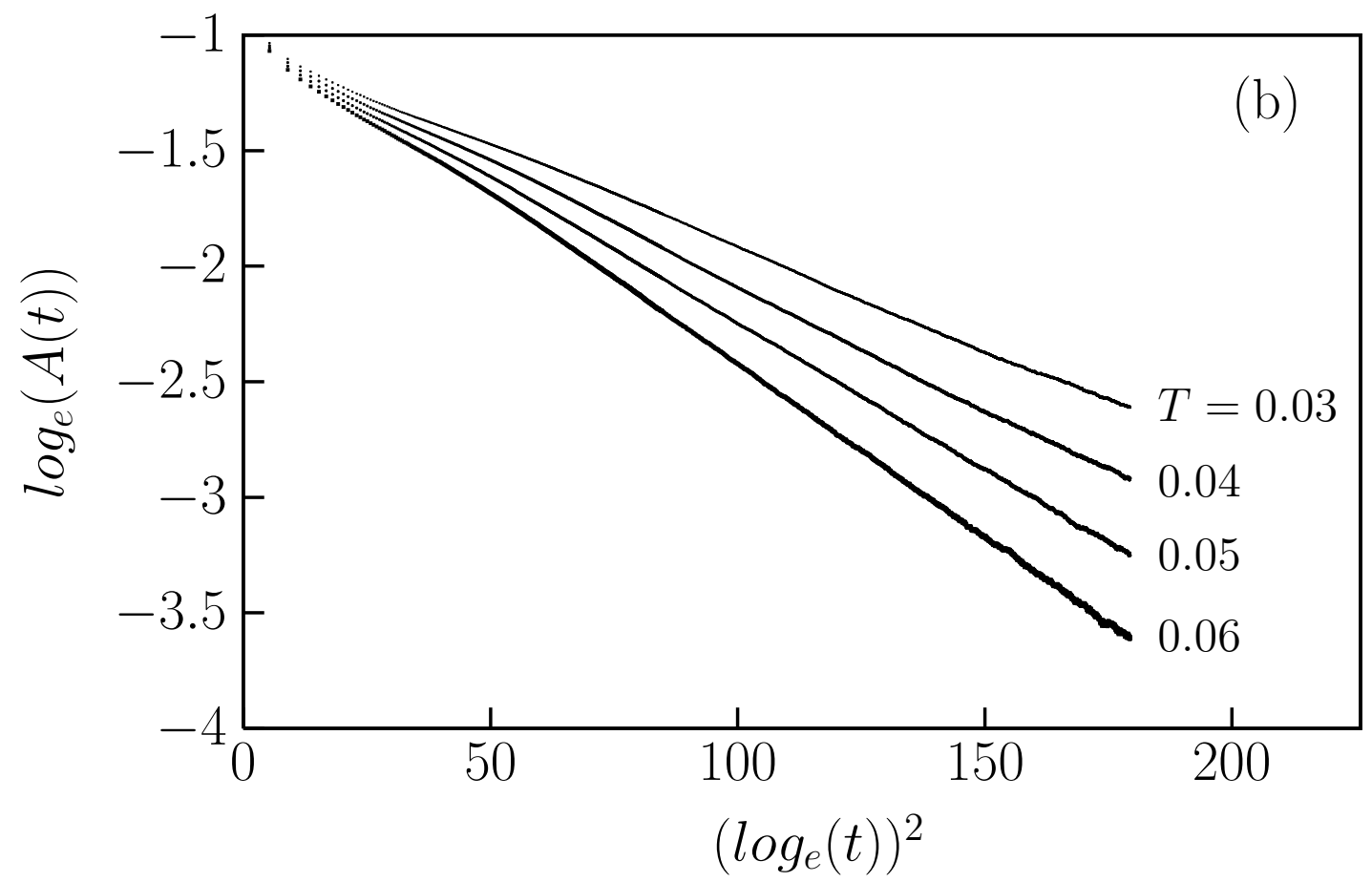

